\title{
Granular flow experiment using artificial gravity generator on International Space Station
}

\section{Shingo Ozaki ( $\nabla$ s-ozaki@ynu.ac.jp )}

Yokohama National University

\section{Genya Ishigami}

Keio University

\section{Masatsugu Otsuki}

Japan Aerospace Exploration Agency

Hirdy Miyamoto

University of Tokyo

\section{Koji Wada}

Chiba Institute of Technology

\section{Yutaro Watanabe}

Yokohama National University

\section{Takaru Nishino}

Yokohama National University

Hiromichi Kojima

Keio University

\section{Keinosuke Soda}

Keio University

\section{Yuki Nakao}

Keio University

Masataku Sutoh

Japan Aerospace Exploration Agency

\section{Takao Maeda}

Japan Aerospace Exploration Agency

\section{Taizo Kobayashi}

Ritsumeikan University

\section{Article}

Keywords: Granular media, artificial gravity generator, mass flow rate, discrete element method, International Space Station

Posted Date: June 21st, 2021 
DOI: https://doi.org/10.21203/rs.3.rs-598295/v1

License: (c) (1) This work is licensed under a Creative Commons Attribution 4.0 International License. Read Full License 


\section{Title}

Granular flow experiment using artificial gravity generator on International Space

Station

\section{Authors}

S. Ozaki ${ }^{1 *}$, G. Ishigami ${ }^{*}$, M. Otsuki ${ }^{3 *}$, H. Miyamoto ${ }^{4}$, K. Wada ${ }^{5}$, Y. Watanabe ${ }^{1}$, T.

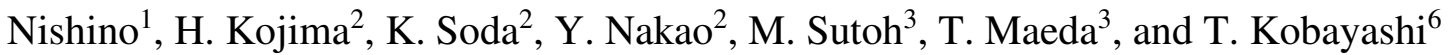

${ }^{1}$ Yokohama National University, ${ }^{2}$ Keio University, ${ }^{3}$ Japan Aerospace Exploration Agency, ${ }^{4}$ The University of

Tokyo, ${ }^{5}$ Chiba Institute of Technology, ${ }^{6}$ Ritsumeikan University, ${ }^{*}$ equally contributed.

1* 79-5 Tokiwadai, Hodogaya-ku, Yokohama 240-8501, Japan. Email: s-ozaki@ ynu.ac.jp

2*3-14-1 Hiyoshi, Yokohama 223-8522, Japan. Email: ishigami@mech.keio.ac.jp.

3* 3-1-1 Yoshinodai Chuo-ku, Sagamihara-shi, Kanagawa 252-5210, Japan. Email: otsuki.masatsugu@ jaxa.jp 


\title{
Granular flow experiment using artificial gravity generator on
}

\section{International Space Station}

\begin{abstract}
Studying the gravity-dependent characteristics of regolith, fine-grained granular media covering extra-terrestrial bodies is essential for the reliable design and analysis of landers and rovers for space exploration. We performed a granular flow experiment under stable artificial gravity conditions generated by a centrifuge on the International Space Station. We also performed a discrete element simulation of the granular flow in both artificial and natural gravity environments. The simulation results verified that the granular flows in artificial and natural gravity are consistent. Further, regression analysis of the granular flow results revealed that the mass flow rate quantitatively follows a well-known physics-based law with some deviations under low-gravity conditions, implying that the bulk density of the granular media decreases with gravity. This insight also indicates that the bulk density considered in simulation studies of space probes under low-gravity conditions needs to be tuned for their reliable design and analysis.
\end{abstract}

Keywords: Granular media; artificial gravity generator; mass flow rate; discrete element method; International Space Station

\section{Introduction}

Technologies for landing and roving on extra-terrestrial bodies are fundamental for pursuing scientific missions and human exploration in space, as reported in the roadmaps published by several space agencies ${ }^{1,2,3}$. Solar rocky bodies, including the Moon and asteroids, are covered by fragmental debris, called regolith. Regolith, which 
is a potentially fluffy, powdery, granular material is a primary concern for the lander or rover; landing on such loose soil is a critical phase during exploration as the footpad of the landing gear may bury into the regolith. Therefore, the mechanical interaction of the footpad plays a key role in the landing dynamics ${ }^{4,5,6}$. The importance thereof is borne out by the fact that one mission involving Spirit, one of the twin mars exploration rovers, had to be terminated because the wheel of the rover became trapped by loose regolith $^{7}$. This issue has clarified the importance of the wheel-soil interaction mechanics ${ }^{8,9}$. These mechanical interaction models of machines (i.e., landing gear or mobility system) on such granular media under various levels of gravitational acceleration are key to their reliable design and analysis. For instance, the pressuresinkage relationship ${ }^{10}$, often seen in a wheel-soil interaction model, considers the weight density of granular media for determining sinkage with respect to the pressure applied. The alternative models proposed in the last decade, such as the empirical resistive force theory ${ }^{11,12,13}$, a local friction force model ${ }^{14}$, a unified force model for the $\operatorname{drag}^{15}$, and a modified Archimedes law ${ }^{16}$, also implicitly include gravitational acceleration because they assume hydrostatic-like force in accordance with the penetration depth of solid objects into granular media.

The discrete element method (DEM) is a numerical approach that precisely simulates the dynamic response of granular media in mechanical interaction ${ }^{17,18}$. In the past, several studies using DEM focused on predicting vehicle mobility in regolith sediments on the Earth, Moon, or Mars ${ }^{19,20,21,22,23}$ as they explored the effect of gravity on sinkage and motion resistance of vehicle wheels. The DEM has also been employed to address granular media in low-gravity environments ${ }^{19,20,24}$.

Such a computer-aided approach often requires a reliable scheme for accuracy evaluation of the simulated output. According to verification-and-validation (V\&V) 
standards ${ }^{25,26}$, any simulation model and its output must be quantitatively evaluated and validated by comparing them with the corresponding experimental results. Therefore, the DEM analysis of granular dynamics in an extra-terrestrial environment also needs benchmark data obtained at the corresponding test environment under varied gravity and high-vacuum conditions to be verified.

The mechanical characteristics of granular media under different gravity conditions have been experimentally investigated, and it was found that their flow dynamics explicitly depend on the gravitational acceleration ${ }^{27}$. The angle of repose is constant regardless of the gravitational acceleration ${ }^{28,29}$, while the friction angle slightly increases as the gravity decreases ${ }^{30}$. The wheel mobility performance under reduced gravity has also been investigated ${ }^{31}$. The abovementioned studies ${ }^{27-31}$ employed parabolic flights or drop tower facilities for simulating reduced gravity environments on Earth. We note that these facilities can simulate only limited campaigns: short duration (e.g., a few tens of seconds in parabolic flights), fewer test trials, and unstable gravity conditions $^{32}$.

While the experimental approach limits the number of trials and gravity conditions, dimensional analysis is a typical approach used for discussing the gravitydependent characteristics of the granular flow. Beverloo's law ${ }^{33}$, the most commonly used law for flow rate analysis of granular media, postulates that the steady-state mass flow rate is proportional to the square root of the gravitational acceleration. The law has been modified for wider outlet sizes ${ }^{34}$ and validated under high-gravity conditions $35,36,37$. However, its applicability under low-gravity conditions is still uncertain.

To address the abovementioned issues, we conducted granular flow experiments under low-gravity conditions by using hourglass-shaped devices in the Japanese Experimental Module (JEM) on the International Space Station (ISS). The JEM is 
equipped with a centrifuge providing high-quality, long-term, and stable artificial gravity (AG) conditions ${ }^{38,39}$. We employed a wide range of $\mathrm{AG}$ levels, from $0.063 \mathrm{G}$ to 2.0 G, over seven hours using several granular media. A DEM simulation study also supported the consistency of the granular dynamics in the AG and natural gravity fields. We found that the mass flow rate of the granular media qualitatively follows Beverloo's law, while some deviations are observed under low-gravity conditions. The statistical and dimensional analysis of the deviation implies that the low gravity decreases the bulk density of the granular media. This outlook leads to feasible insight for designing and analysing the space probes under low-gravity conditions.

\section{Results and discussion}

\subsection{Experiment}

The centrifuge (Fig. 1(a) and (b)) in the experiment is used to rotate the experimental apparatus (Fig. 1(c) and (d)) with arbitrary angular velocity to obtain AG range of 0.063-2.0 G with specific gravity steps every 13 min (Fig. 1(e)). Table 1 shows snapshots after the flow under microgravity $(0.063 \mathrm{G})$ along with microphotographs, and mass packed in the hourglass. Note that the inside of each hourglass is vacuum. The typical properties of the granular media, such as particle size distribution, shear strength, and particle and bulk densities, are available in Supplementary Text 1 . The experimental campaign continued for seven hours. Supplementary Text 2 describes the geometric layout of the experimental apparatus (specifications described in Supplementary Text 3) and angular velocity of the centrifuge according to varied AG levels. The acceleration profile of each AG is summarised in Supplementary Text 4. The Data Availability statement provides the raw movies (MP4 files) of the flows of the granular media. 
Among the granular media listed in Table 1, the alumina beads, silica sand No. 5, and Toyoura sand exhibited explicit mass flow with some periodic intermittency effects $^{40}$, particularly observed under the condition of relatively low gravity (see Data Availability section). By contrast, the other materials flew with multiple modes such as mass/funnel flows ${ }^{41,42,43}$ that vary along with the levels of gravity conditions. In addition, these granular media adhered, deposited, or even aggregated in the hourglass. This difference may be due to the particle size and its distributions, as discussed below.

Figure 2 illustrates the relationship between the particle size and gravitational forces (particle weight) and Van der Waals force of a particle. The median diameter (D50) of the granular media is indicated as the open-circle plot along with the minimum and maximum particle range of the granular media. Assuming a spherical particle, the effective particle weight due to gravity is proportional to the cube of the particle size $x$. In contrast, the Van der Waals force (alumina with a smooth surface) is proportional to $x$.

Figure 2 implies that the Van der Waals force is dominant for particles smaller than approximately $50 \mu \mathrm{m}$ even under $1.0 \mathrm{G}$. In particular, the granular media (\#04 to \#08, Table 1) contain a large number of fine particles, resulting in a remarkable effect of adhesive forces. Furthermore, because of the repeated flow by flipping the hourglass, the fine particles collide with one other or with the walls under vacuum conditions. An electrostatic force may be generated owing to the charged particles, which is beyond the scope of this study. Nevertheless, we deduce that this force may excite the particle adhesion to the hourglass wall made of borosilicate glass. These factors impede the explicit flow of the granular media of \#04 to \#08, as shown in the snapshots of Table 1.

In contrast, the particle weight due to gravity is the dominant factor for particles larger than about $200 \mu \mathrm{m}$ even in microgravity $(0.063 \mathrm{G})$. The granular media, alumina 
beads, silica sand No. 5, and Toyoura sand, are above this threshold, showing explicit granular flows under varying gravity levels. Thus, the following analysis focuses on these three granular media to discuss the gravity-dependent characteristics of granular flow.

Figure 3 shows the mass flow rates under different AG levels. The mass flow rates of all granular media are proportional to the square root of the AG regardless of the hopper angles. The granular flows at the hopper angle of $60^{\circ}$ are faster than those at $120^{\circ}$. This is because the steeper the slope is towards the hourglass orifice, the higher is the flow rate ${ }^{44}$. Among the three granular media, the alumina beads have a relatively high mass flow rate because of their spherical particle shapes with a uniform diameter. Silica sand No. 5 and Toyoura sand show similar characteristics for their mass flow rate with a slight difference (a few grams per second) owing to their similar particle size distributions.

\subsection{Discrete element method analysis}

The DEM analysis was performed to examine the difference between the AG environment on the ISS and the natural gravity (NG) environment. In the former, the experiment on the ISS was mimicked, i.e., the hourglass was repeatedly flipped while being revolved (see Supplementary Movies 1 and 2). However, in the latter, a prescribed uniform gravitational field was applied to granular media, and only repeated flipping of the hourglass was performed.

Figure 4 shows a snapshot captured during the flow of alumina beads under 0.1 G. Details on the DEM parameters and conditions are provided in the Methods section. It can be confirmed that in the AG environment, the particles fall towards the rearward direction deviating from the centrifugal force direction after passing through the orifice. 
The reason is that the falling particles are in the inertial coordinate system, and when observing them from the hourglass in the rotating coordinate system, the Coriolis force induces particles to move in the tangential direction of the rotating orbital. The granular flow therefore deviates towards the $+y$ direction (thickness direction) of the hourglass. However, for AG, the sedimentary particles in the upper section of the hourglass are in the rotating system just like the hourglass. Therefore, the effects of gravity condition on the flow mode in the upper box, such as mass flow and funnel flow, are considered to be similar to those of NG.

Figure 3 also includes the gravity dependence of the mass flow rate obtained via the DEM analysis for alumina beads, silica sand No. 5, and Toyoura sand. We confirmed that the DEM analytical results show good agreement with the experimental results. Thus, we could examine the effects of the gravitational environment on the flow rate.

Figure 5 shows the gravity dependence of the particle velocity obtained by DEM analyses for alumina beads, silica sand No. 5, and Toyoura sand. Here, the particle velocity was measured at the central position just below the orifice and was evaluated as the averaged velocities of passing particles. For the detail of the measured data, see Supplementary Text 5. The open plots and closed plots correspond to the NG and AG conditions, respectively. Because the effect of the Coriolis force on the flow rate immediately after the fall is small (falling distance is less than $1 \mathrm{~mm}$ ), the results of gravity-dependency are almost the same under both gravity conditions, especially in the case of a hopper angle of $120^{\circ}$ or of low gravity level. Meanwhile, when the hopper angle is $60^{\circ}$ and a high gravity level is employed, the magnitude of the average velocities is different between the NG and AG conditions. However, it is noteworthy 
that the average particle velocities of all the granular media are still proportional to the square root of the gravity regardless of the gravity conditions.

Figure 6(a) shows the calculation results of the trajectories of the particles as seen from the $y-z$ plane of the hourglass under the AG condition. Here, we used the initial velocities of the particles obtained via the DEM analyses under the AG condition (Fig. 5) as those for trajectory calculation. Because the angular velocity of the rotary table changes according to the magnitude of the AG, similar trajectories are seen regardless of the AG level within the range of geometric conditions of the present experiment. The particles on the camera side do not collide with the back wall during the fall (Fig. 4).

Figure 6(b) shows the ratio between the velocities at the bottom collision to the initial falling velocity immediately below the orifice. The magnitude of the velocities at the bottom was evaluated by considering a constant acceleration motion in the NG environment and revolution condition in the AG environment. Here, the maximum drop height in the hourglasses is $21 \mathrm{~mm}$ (see Fig. 7). We confirmed that the particle velocities at the bottom collision are higher than the initial velocities under both the AG and NG conditions. Moreover, the effect of Coriolis force for the change in velocity is smaller than the effect of gravitational acceleration, which means that the Coriolis force has little effect even on the disturbance of sedimentary state in the present experimental condition.

These observations collectively indicate that, while the apparent flow (fall) direction in the $\mathrm{AG}$ environment deviates from that of the $\mathrm{NG}$ environment with the falling time, our experiment is appropriate to investigate the dependence of gravity on the granular flow dynamics. In particular, for granular media \#4-\#8 including fine particles, the effects of adhesive forces on the granular flow under low-gravity 
conditions are interesting ongoing issues as its competition with gravity affects the flow-mode transitions, such as mass/funnel flows or bridging.

In addition, as the particles fallen at the bottom belong to the rotating system again, it could also be possible to examine the gravity dependence on static sedimentary phenomena, including the angle of repose and bulk density. Furthermore, the data obtained by the present experiment for eight types of granular media might be used for parametric fitting and V\&V of the DEM model under low-gravity conditions. Then, the data can be applied to simulations of more complicated dynamics of granular media and interaction phenomena with solid objects. Note that, in such future work, it is necessary to introduce a Van der Waals force and/or electrostatic force on the DEM analysis model.

\subsection{Statistical analysis and outlook}

The experimental results are discussed based on Beverloo's law ${ }^{33}$, which postulates the mass flow rate of granular media $\left(\hat{v}_{m}\right)$ in the steady state as

$$
\hat{v}_{m}=C_{f} D p_{b}\left(\xi g_{0}\right)^{0.5},
$$

where $C_{f}$ is the discharge coefficient, $p_{b}$ is the bulk density of the granular media, $g_{0}$ is the standard gravity acceleration $\left(9.80665 \mathrm{~m} / \mathrm{s}^{2}\right)$, and $D$ denotes the variables determined by the orifice diameter and the particle diameter. $\xi$ is the gravity magnitude generated by the centrifuge; in other words, it is the scaling factor of the gravity acceleration with regard to standard gravity.

Figure 8 summarises the regression analysis results of the mass flow rate. The regression curve is based on the nonlinear regression function as follows:

$$
v_{m}=\alpha \xi^{0.5+\beta}
$$


When comparing Eqs. (1) and (2), the coefficient $\alpha$ is constant regardless of the gravity level, namely $\alpha=C_{f} D \rho_{b} g_{0}^{0.5}$. The coefficient $\beta$ indicates how the experimental result deviates from the rule of the square root of gravity. Table 2 summarises the values of these coefficients determined from the analysis, which focused on the gravity magnitudes in the range of $0.0-1.0 \mathrm{G}$ because we have sufficient datasets in those ranges. The value of $\alpha$ depends on the particle shape, diameter, density, and frictional property of the granular media. The absolute value of $\beta$ is less than 0.1 , and the coefficients of determination are approximately 0.9687 or higher. The experiment quantitatively well follows Beverloo's law.

Further outlook can be discussed based on the deviation between the experiment and Beverloo's law due to the value of $\beta$. Equations (1) and (2) can be combined as follows:

$$
v_{m}=C_{f} D \rho_{b}\left(\xi g_{0}\right)^{0.5} \cdot \xi^{\beta}
$$

Here, the last term $\xi^{\beta}$ can be assumed as the correction coefficient for the law. For a positive $\beta$, the coefficient becomes larger as the scale factor for the gravity acceleration increases. Accompanying the bulk density to the correction coefficient, namely $\rho_{b}^{\prime}=\rho_{b} \xi^{\beta}$, implies that the corrected bulk density $\rho_{b}^{\prime}$ decreases as the gravitational acceleration decreases. This assumption intuitively agrees with the fact that the granular media falling under lower gravity acceleration accumulate with larger void spaces, resulting in a smaller bulk density of granular media. In other words, the porosity of the granular media increases under lower gravity accelerations. Therefore, the correction coefficient $\xi^{\beta}$ may be assumed as a gravity-dependent characteristic for the bulk density of the granular media. 
The abovementioned phenomenon will be useful for the design and analysis of the space probe. For example, the bulk density used in the mechanical interaction model or simulation needs to be tuned such that the density decreases as the gravity decreases.

\section{Methods}

\subsection{Materials}

In this experiment, the eight types of granular media shown in Table 1 were used.

Alumina beads were adopted as the comparative material, which has almost-spherical particles and a considerably narrow particle size distribution. To enable an image analysis, $30 \%$ of packed alumina beads were coloured black using a permanent marker. In addition, as typical sandy soils for which many soil test results have been reported, silica sand (Tohoku sand) No. 5 and No. 8 and Toyoura sand were adopted ${ }^{45,46,47}$. Furthermore, considering the use for future space exploration, four types of regolith simulants, including Lunar regolith simulant ${ }^{31}$ and Phobos regolith simulant ${ }^{48}$, were adopted. The details of the soil test results, such as regarding the particle size distribution, shear strength obtained using a direct shear test, particle and bulk density tests, and static friction test, are summarised in Supplementary Text 1.

\subsection{Experimental apparatus}

The experimental apparatus (Fig. 7(a)) consists of the hourglass-shaped device, CPU board with a camera module, servo motor, accelerometer, and battery packs whose specifications are listed in Supplementary Text 3. The hourglass (Fig. 7(b)) is composed of aluminium alloy, and its front and back sides are covered by borosilicate glass. The hourglass packs a certain amount of granular material in vacuum of less than $20 \mathrm{~Pa}$. The hourglass has two different hopper angles $\left(60^{\circ}\right.$ and $\left.120^{\circ}\right)$. The servo motor flips the 
hourglass $180^{\circ}$ with respect to the artificial gravitational direction every minute so that we can observe granular flow from the different hopper angles in every hourglass flip, where the flip takes about $0.6 \mathrm{~s}$ to complete. Note that the rotation range (flipping motion) is limited to $\pm 90^{\circ}$ because of the size constraints of the experimental apparatus.

The CPU with the onboard camera grabs the flow images at $25 \mathrm{fps}$ and stores them in a compressed MP4 format (H.264, $40 \mathrm{Mbps})$ file every five minutes. These images are resampled from MP4 files at a constant frame rate and undistorted using a calibration template calculated at the pre-flight check. Each image is then correlated with the gravity condition generated by the AG.

The mass flow rate $v_{m}$ is calculated from the experiment as:

$$
v_{m}=\frac{m}{t_{\mathrm{e}}-t_{0}}
$$

where $m$ is the mass of the granular media packed in the hourglass; $t_{0}$ is the time when the flow starts, which is equivalent to the moment when the hourglass is perpendicular to the AG direction during its flip; and $t_{\mathrm{e}}$ is the time when particles do not move among the consecutive images. The temporal resolution is $0.04 \mathrm{~s}$, which is determined by the camera frame rate $(25 \mathrm{fps})$.

\subsection{Discrete element method}

The DEM is a method in which the analytical object is modelled as an aggregate of discrete particles, and their motions at each instant is analysed considering contact and sliding between the particles or particle and object ${ }^{17,18}$. In this study, the commercial software package Rocky DEM ${ }^{49}$ was used.

In the analyses, each granular media was represented by an aggregate of spherical particles according to their particle size distributions (see Supplementary Text 1). The Hertzian spring-dashpot and Mindlin-Deresiewicz models were adopted for the normal 
and tangential force models, respectively. Further, to express the effect of actual particle shape, the rolling resistance model (type C) was also introduced ${ }^{50}$. Rolling resistance is considered when a moment opposing the rolling motion of a particle is introduced into the modelling. This moment is usually incorporated for practical representation of the effect of non-sphericity on rolling spheres or the effect of surface irregularities on other types of particles.

Table 3 shows the parameters of alumina beads, silica sand No. 5, and Toyoura sand for the present analysis. Here, these parameters were determined with reference to the results of minimum density test and the residual strength obtained by a direct shear test, in addition to the hourglass experiment. Friction coefficients between granular media and diagonal wall of hourglass were obtained using a static friction test (see Supplementary Text 1).

The analysis assuming the NG environment was performed by setting a uniform gravity field and giving only flips to the hourglass. Meanwhile, in the analysis assuming the AG environment in the ISS, both the revolution corresponding to the rotary table and the flips were given to the hourglass under the boundary condition of a zero-gravity field. Here, the centre and radius of the revolution and respective angular velocities were set to the same values as those of the experiment (see Supplementary Text 2). Note that, for the analysis in Fig. 5, the gravitational accelerations under NG conditions were adjusted to the gravity at the centre of the orifice under the AG conditions.

\section{Data availability statement}

The authors confirm that the data supporting the findings of this study are available within the supplementary materials. Further, the representative MP4 data and raw data including soil properties are available via the Open Science Framework at 
https:/osf.io/3zcm2/.

\section{Acknowledgement}

This work is financially supported by Institute of Space and Astronautical Science and Human Spaceflight Technology Directorate, JAXA. The authors acknowledge C. Kurosawa, H. Kasahara, Y. Kawai, T. Sakashita, S. Doi, H. Uchikawa, and T. Mano for their support and management in pursuing the experiments in ISS. The authors would also like to thank K. Sakamoto for his support in manufacturing and assembling the experimental apparatus.

\section{Author contributions}

SO, GI, and MO contributed equally to this work, including the conceptualisation and designed the experiments, analysis, and writing the paper; HM created regolith simulants; KW contributed to the conceptualisation and preparation of the granular media; YW and TN performed the DEM analysis; HK, KS, and YN analysed image processing and mass flow rate; MS and TM developed the hourglass-shaped device; TK performed soil tests for granular media and provided their detailed data.

\section{Competing interests}

The authors declare no competing interest.

\section{References}

1. 2020 NASA Technology Taxonomy, https://techport.nasa.gov/view/taxonomy (as of May 2021).

2. Lange, C. et al. GER Technology Development Map - a coordinated analysis of 
technology development interests. AIAA SPACE 2013 Conf. and Exposition, AIAA 2013-5505 (2013).

3. ISECG Technology Working Group. Global exploration roadmap critical technology needs. (2019).

4. Rogers, F. Apollo experience report - Lunar module landing gear subsystem. NASA TN D-6850 (1972).

5. Wan, J., Nie, H., Chen, J., \& Lin, Q. Modeling and simulation of lunar lander softlanding using transient dynamics approach. Proc. of the 2010 Int. Conf. on Computational and Information Sciences, pp. 741-744 (2010).

6. Nohmi M. \& Miyahara, A. modeling for lunar lander by mechanical dynamics software. AIAA Modeling and Simulation Technologies Conf. and Exhibit, AIAA 2005-6416 (2015).

7. Kerr, R. Mars rover trapped in sand, but what can end a mission? Science, 324, 998 (2009).

8. Ishigami, G., Miwa, A., Nagatani, K. \& Yoshida, K. Terramechanics-based model for steering maneuver of planetary exploration rovers on loose soil. J. Field Robotics, 24, 233-250 (2007).

9. Ding, L. et al. Interaction mechanics model for rigid driving wheels of planetary rovers moving on sandy terrain with consideration of multiple physical effects. $J$. Field Robotics, 32, 827-859 (2015).

10. Wong, J. Theory of ground vehicles. Fourth edition (John Wiley \& Sons Inc., New Jersey, 2008). 
11. Li, C., Zhang, T. \& Goldman, D. A terradynamics of legged locomotion on granular media. Science 399, 1408-1412 (2013).

12. Askari, H., \& Kamrin, K. Intrusion rheology in grains and other flowable materials. Nature Mater. 15, 1274-1279 (2016).

13. Shrivastava, S. et al. Material remodeling and unconventional gaits facilitate locomotion of a robophysical rover over granular terrain. Sci. Rob. 5, eaba3499 (2020).

14. Brzinski III, T., Mayor, P. \& Durian. D. Depth-dependent resistance of granular media to vertical penetration. Phys. Rev. Lett. 111, 168002 (2013).

15. Meer, van der D. Impact on granular beds. Annu. Rev. Fluid Mech. 49, 463-484 (2017).

16. Kang, W., Feng, Y., Liu, C. \& Blumenfeld, R. Archimedes' law explains penetration of solids into granular media. Nature Comm. 9, 1101 (2018).

17. Cundall, P.A. A discrete numerical model for granular assemblies. Geotechnique 29, 47-65 (1979).

18. Munjiza, A.A. The combined finite-discrete element method. (John Wiley \& Sons Ltd., Chichester, UK, 2004).

19. Nakashima, H. et al. Discrete element method analysis of single wheel performance for a small lunar rover on sloped terrain. J. Terramech. 47, 307-321 (2010).

20. Knuth, M., Johnson, J., Hopkins, M., Sullivan, R. \& Moore, J. Discrete element modeling of a Mars Exploration Rover wheel in granular material. J. Terramech. 
49, 27-36 (2012).

21. Nakashima, H. \& Kobayashi, T. Effects of gravity on rigid rover wheel sinkage and motion resistance assessed using two-dimensional discrete element method. $J$. Terramech, 53, 37-45 (2014).

22. Johnson, J.B. et al. Discrete element method simulations of Mars Exploration Rover wheel performance, J. Terramech, 62, 31-40 (2015).

23. Johnson, J.B., Duvoy, P.X., Kulchitsky, A.V., Creager, C., \& Moore, J. Analysis of Mars Exploration Rover wheel mobility processes and the limitations of classical terramechanics models using discrete element method simulations. J. Terramech, 73, 61-71 (2017).

24. Opsomer, E., Noirhomme, M., Vandewalle, N., Falcon, E. \& Merminod, S. Segregation and pattern formation in dilute granular media under microgravity conditions. NPJ Microgravity 3 (2017).

25. ASME, V\&V 10, Guide for verification and validation in computational solid mechanics (2006).

26. NASA STD 7009, Standard for models and simulations (2006).

27. Reiss, P., Hager, P., Hoehn, A., Rott, M. \& Walter, U. Flowability of lunar regolith simulants under reduced gravity and vacuum in hopper-based conveying devices. $J$. Terramech. 55, 61-72 (2014).

28. Nakashima, H. et al. Determining the angle of repose of sand under low-gravity conditions using discrete element method. J. Terramech. 48, 17-26 (2011). 
29. Marshall, J. et al. Failures in sand in reduced gravity environments. J. Mech. \& Phys. of Solids 113, 1-12 (2018).

30. Karapiperis, K., Marshall, J. \& Andrade, J. Reduced gravity effects on the strength of granular matter: DEM simulations versus experiments, J. Geotech. \& Geoenv. Eng. 146, 06020005 (2020).

31. Kobayashi, T., Fujiwara, Y., Yamakawa, J. \& Yasufuku, N. Mobility performance of a rigid wheel in low gravity environments. J. Terramech. 47, 261-274 (2010).

32. Carr, C.E., Bryan, N.C., Saboda, K.N. et al. Acceleration profiles and processing methods for parabolic flight, NPJ Microgravity 4, 14 (2018).

33. Beverloo, W.A., Leniger, H.A. \& Van de Velde, J. The flow of granular material through orifices. J. Chem. Eng. Sci. 15, 260-296 (1961).

34. Mankoc, C. et al. The flow rate of granular materials through an orifice, Granul. Matt. 9, 407-414 (2007).

35. Le Pennec, T. et al. Effect of gravity on mass flow rate in an hour glass. Powder Technol. 85, 279-281 (1995)

36. Dorbolo, S. et al. Influence of the gravity on the discharge of a silo. Granul. Matt. 15, 263-273 (2013).

37. Barbir, O. \& Mathews, J. Investigation of the influence of gravity on granular flow using silo centrifuge model, 25th European Young Geotechnical Engineers Conference (2016).

38. CBEF : Experiment - International Space Station - JAXA, 
https://iss.jaxa.jp/en/kiboexp/pm/cbef/ (as of May 2021).

39. Morita, H. et al. Feasibility of a short-arm centrifuge for mouse hypergravity experiments. PLoS One 10, e0133981 (2015).

40. Aider, J.-L., Sommier, N., Raafat, T., \& Hulin, J.-P. Experimental study of a granular flow in a vertical pipe: A spatiotemporal analysis. Phys. Rev. E, 59, 778786 (1999).

41. Cizeau, P., Makse, H. \& Stanley, H. Mechanisms of granular spontaneous stratification and segregation in two-dimensional silos. Phys. Rev. E 4408-4421 (1999).

42. Saleh, K., Golshan, S. \& Zarghami, R. A review on gravity flow of free-flowing granular solids in silos - Basics and practical aspects. Chem. Eng. Sci. 192, 10111035 (2018).

43. Rabinovich, E., Kalman, H. \& Peterson, P. Granular material flow regime map for planar silos and hoppers. Powder Technol. 377, 597-606 (2021).

44. Nguyen, T. V., Brennen, C. \& Sabersky R. H. Gravity flow of granular materials in conical hoppers. ASME. J. Appl. Mech. 46(3), 529-535 (1979).

45. Iwasaki, T., Tatusoka, F. \& Takagi, T. Shear moduli of sands under cyclic torsional shear loading. Soils and Foundations 18, 39-56 (1978).

46. Oztoprak, S. \& Bolton, M.D. Stiffness of sands through a laboratory test database. Geotechnique 63, 54-70 (2013).

47. Arshad, M.I., Tehrani, F.S., Prezzi, M. \& Salgado, R. Experimental study of cone 
penetration in silica sand using digital image correlation. Geotechnique 64, 551569 (2014).

48. Miyamoto et al., Surface Environment of Phobos and Phobos Simulant UTPS, Earth Planet. Sci. (in press).

49. ESSS, DEM technical manual, Rocky 4.3, 2020.

50. Ai, J., Chen, J. F., Rotter, J. M. \& Ooi, J. Y. Assessment of rolling resistance models in discrete element simulations. Powder Technol. 206, 269-282 (2010). 


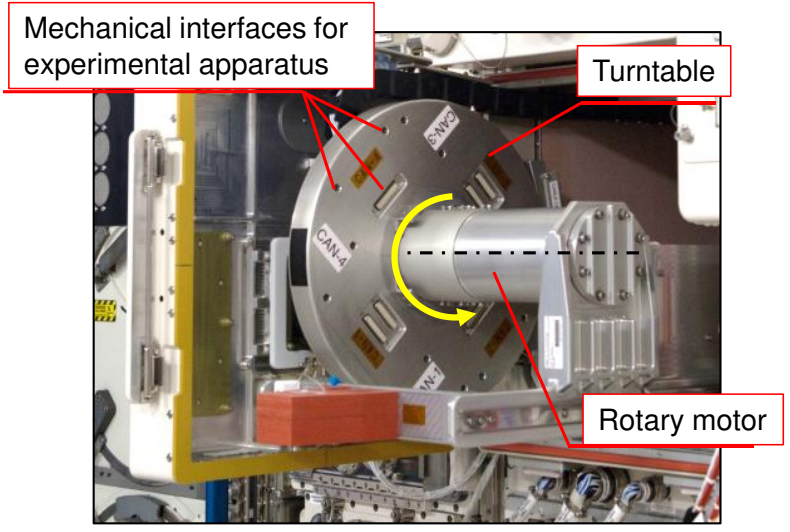

(a)

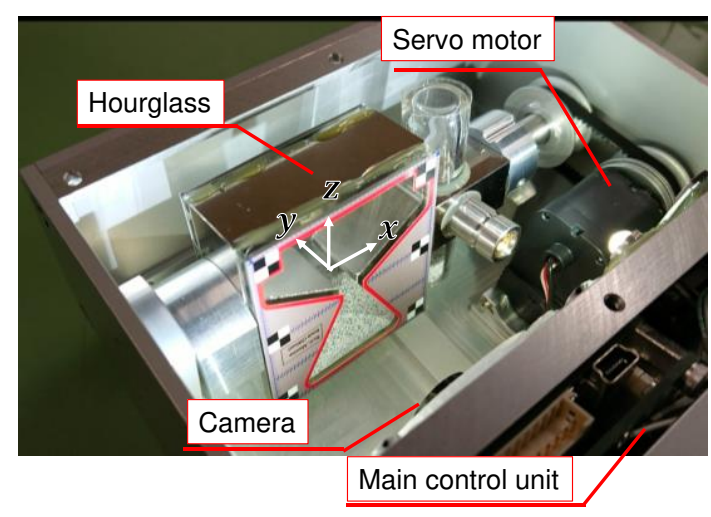

(c)

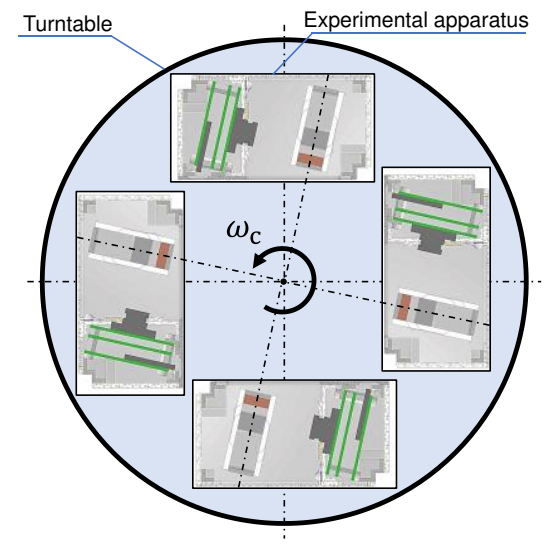

(b)

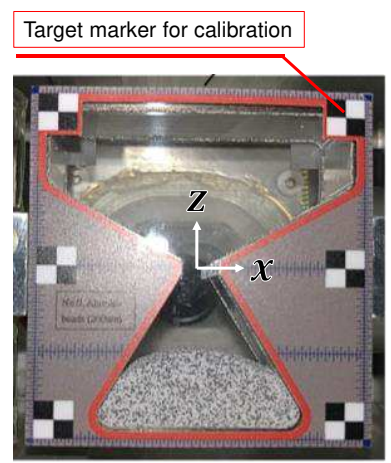

(d)

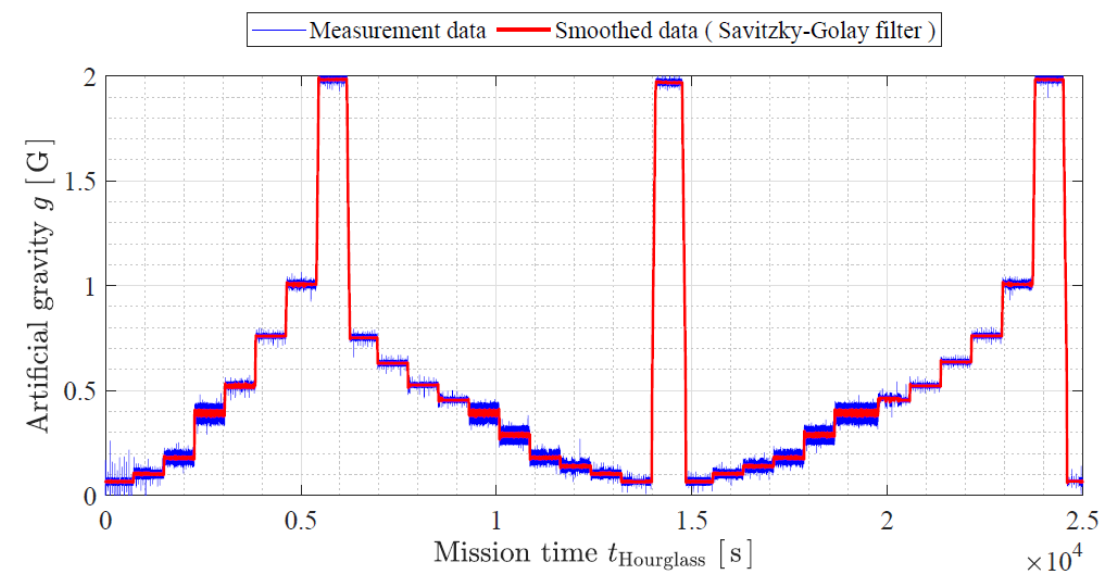

(e)

Figure 1: Overview of the experimental setup, apparatus, and time history of the AG profiles: (a) Centrifuge installed on the JEM/ISS (courtesy JAXA/NASA) without 
attaching the experimental apparatus; (b) Schematic illustration of the centrifuge with the experimental apparatus. The centrifuge installs four sets of the apparatus in each experimental run. We performed two experimental runs for eight sets in total; (c) Snapshot of the experimental apparatus (top cover is removed). The reference coordinate of the hourglass is located on the centre of the orifice; (d) Hourglass image taken by the camera (trimmed image); and (e) Time history of AG in the experimental run ranging over the values $0.063,0.10,0.17,0.38,0.50,0.75,1.0$, and $2.0 \mathrm{G}$. 


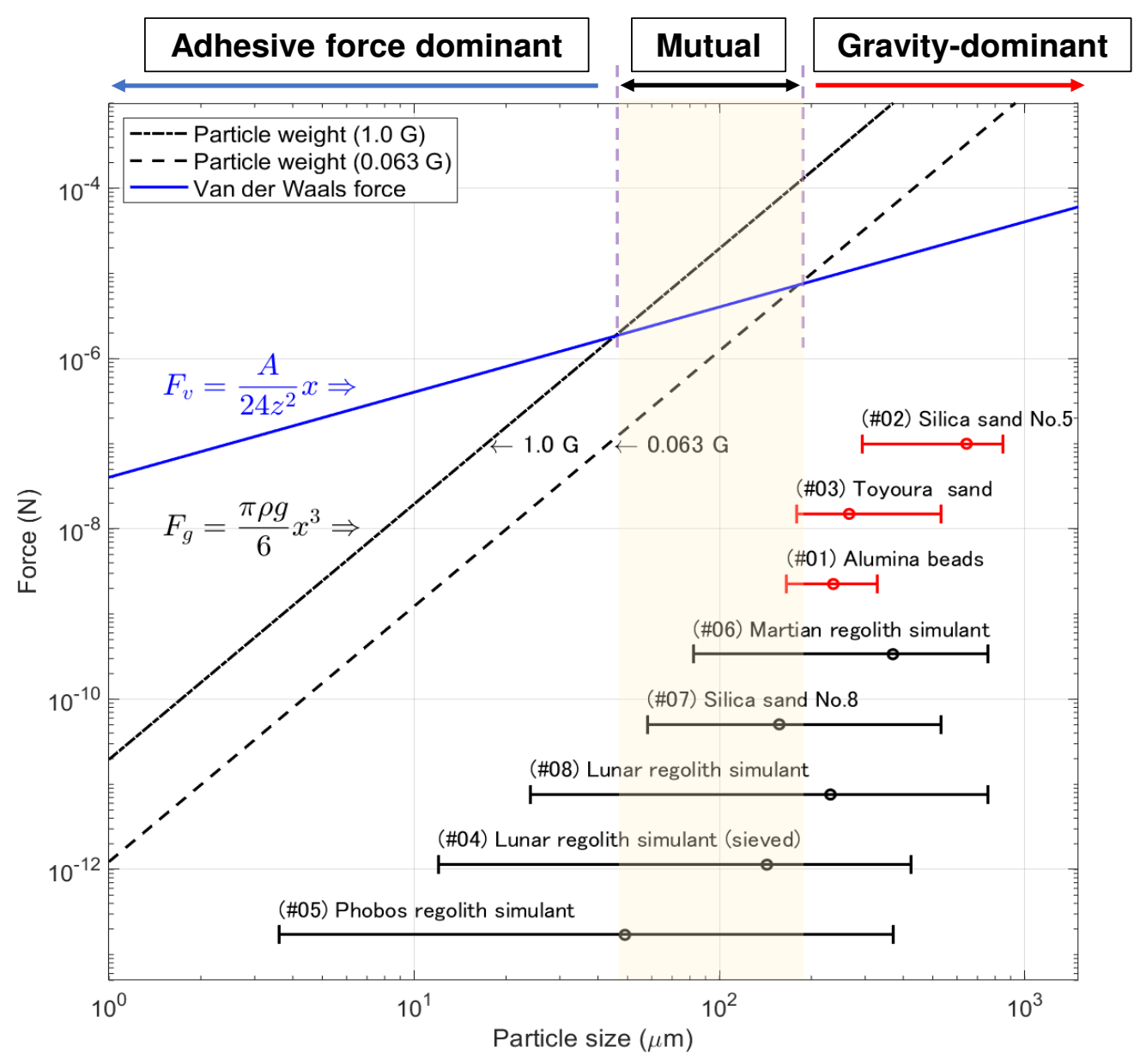

Figure 2: Relationship of particle size $x$, particle weight, and Van der Waals force. The particle is assumed to have a spherical shape and density $\rho$ for the evaluation of forces. The Van der Waals force is estimated under assumptions of the alumina composition without the surface roughness. The parameters of the Van der Waals force are as follows: Hamaker constant $A=15.5 \times 10^{-20} \mathrm{~J}$, and $z=0.4 \mathrm{~nm}$. The horizontal error bars in the graph show the range of particle sizes of each tested granular media. The open circle is the median diameter, and both ends indicate the minimum and maximum particle ranges. The vertical location of the error bars is arbitrary. 


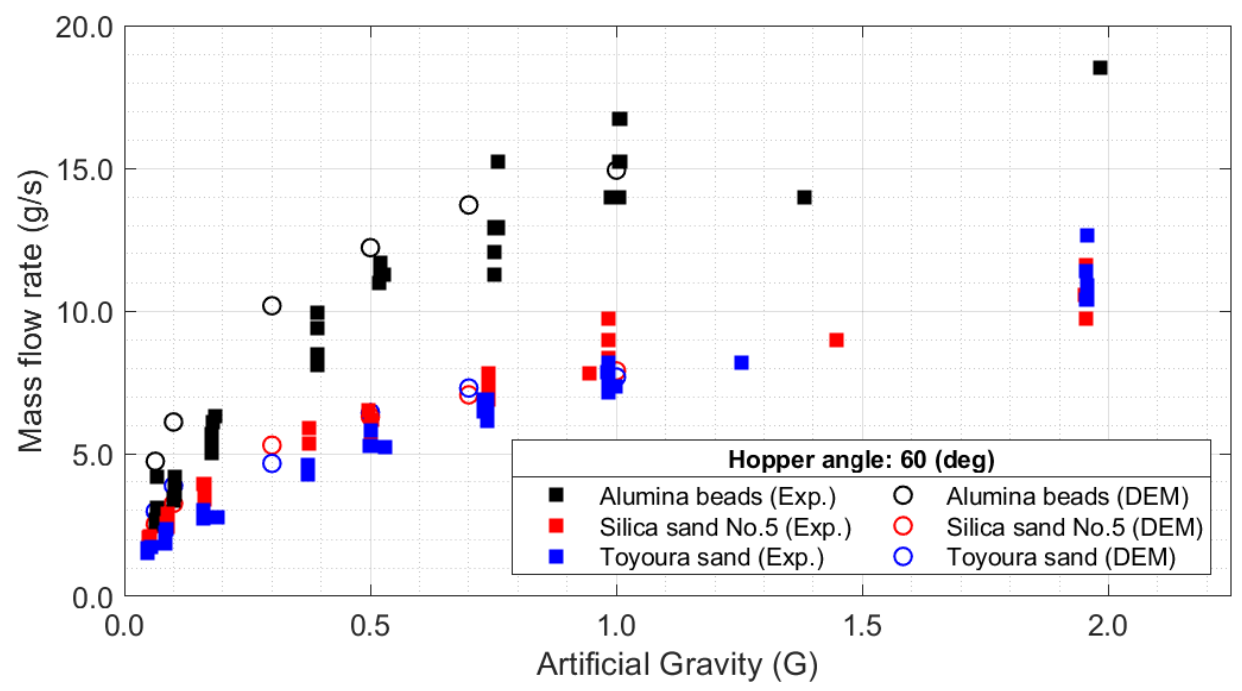

(a)

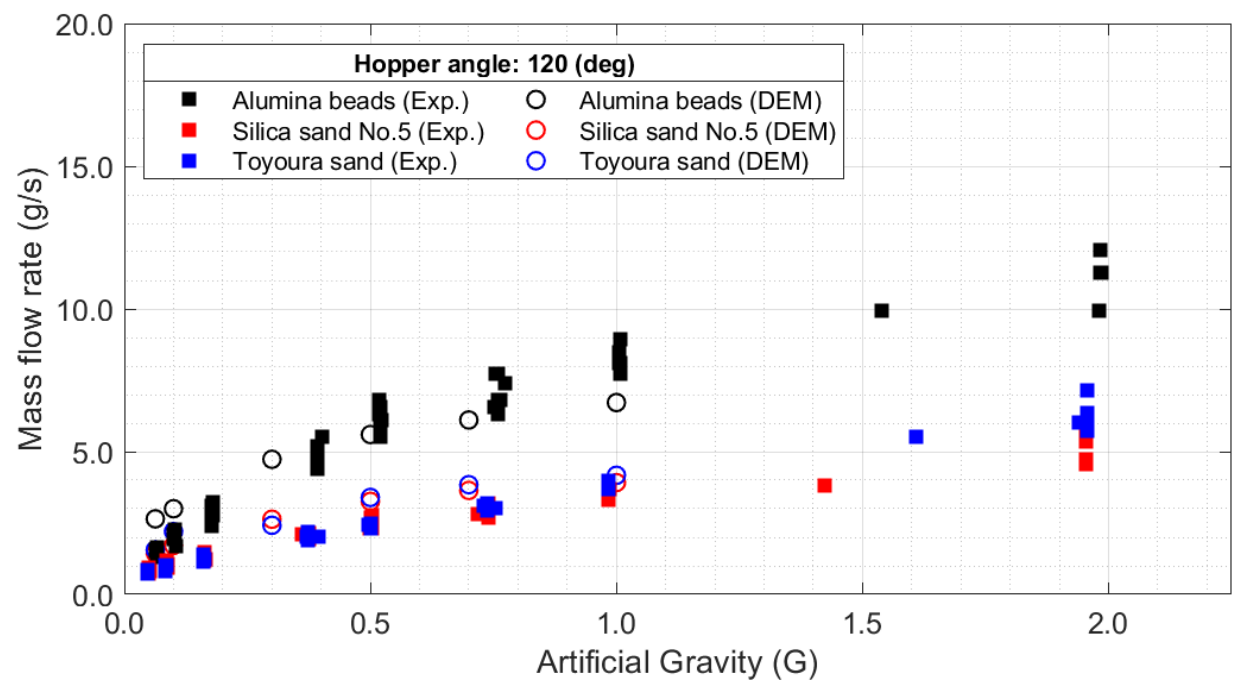

(b)

Figure 3: Mass flow rates of granular media in varied AG levels. The results were obtained for hopper angles of (a) $60^{\circ}$ and (b) $120^{\circ}$. 

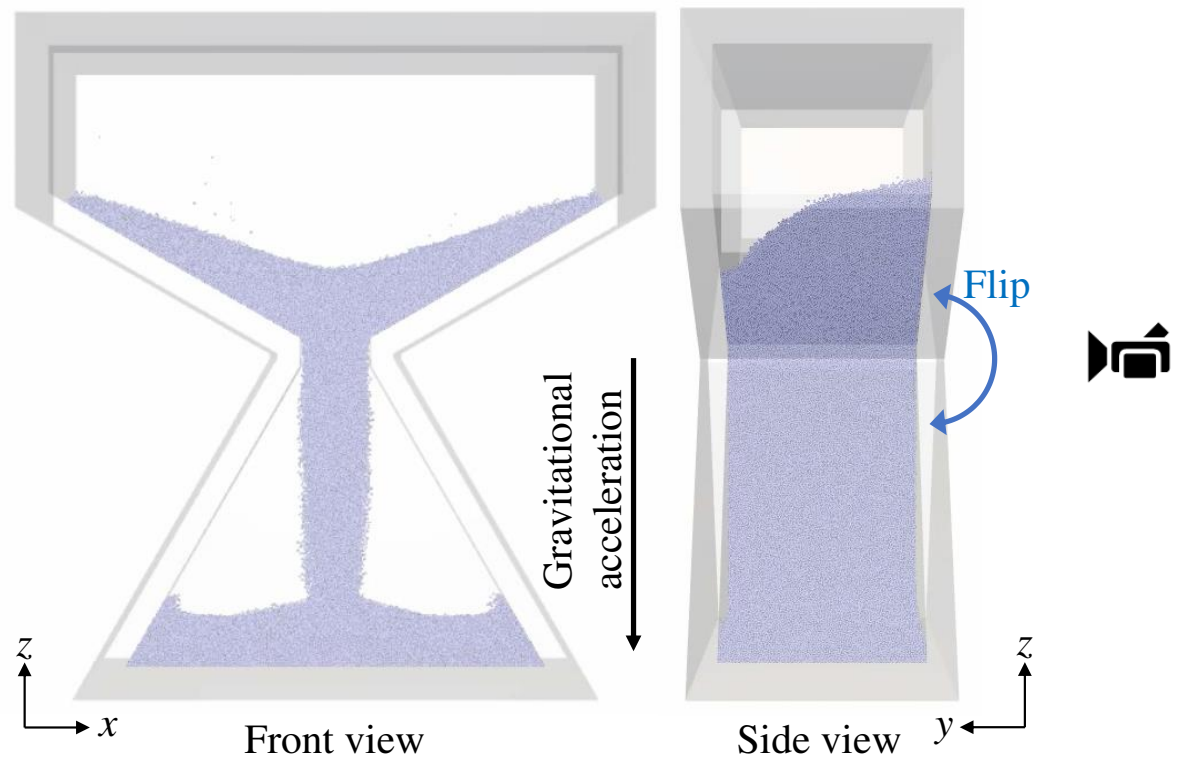

(a)
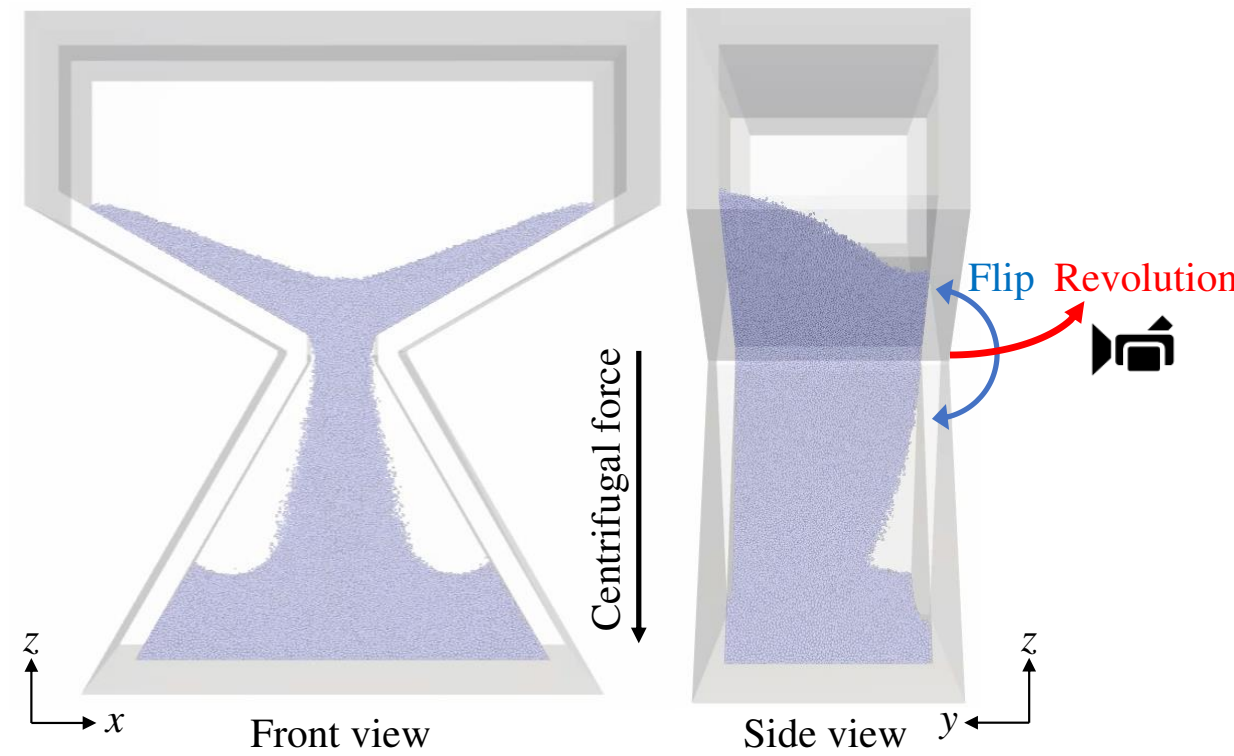

(b)

Figure 4: Snapshots of DEM analysis results of alumina beads at $0.1 \mathrm{G}$ : (a) NG environment; (b) AG environment in the ISS. The front view of the hourglass corresponds to the right-hand side in the side view. 


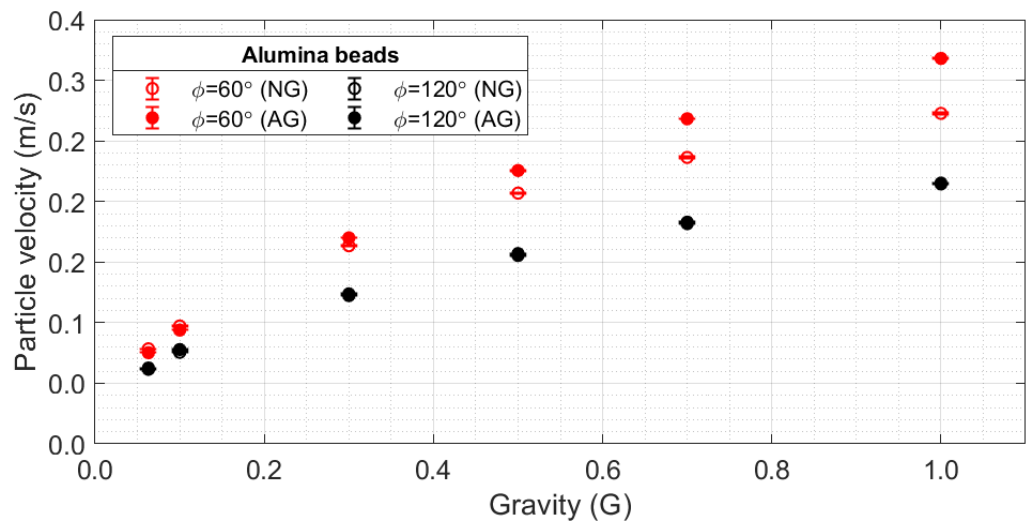

(a)

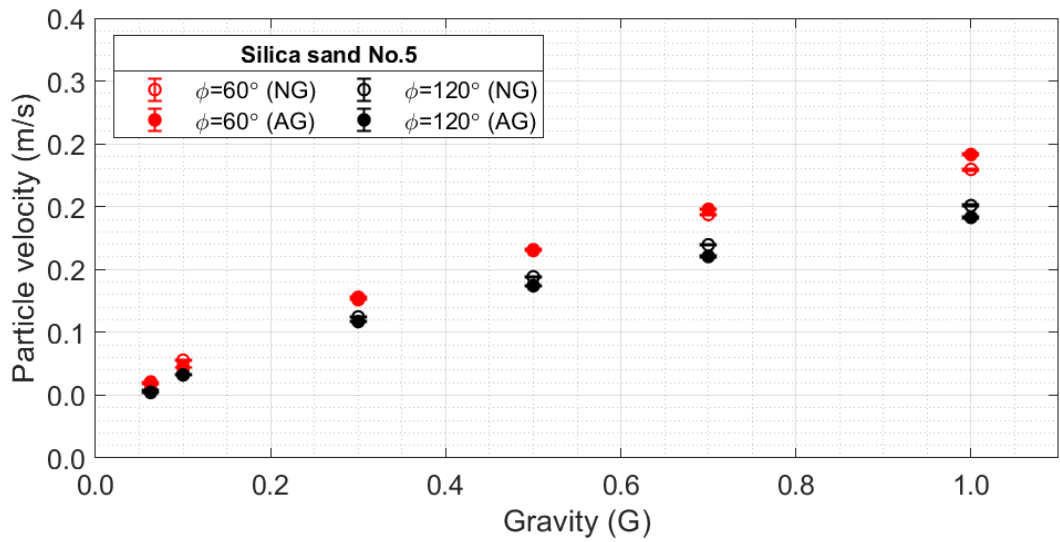

(b)

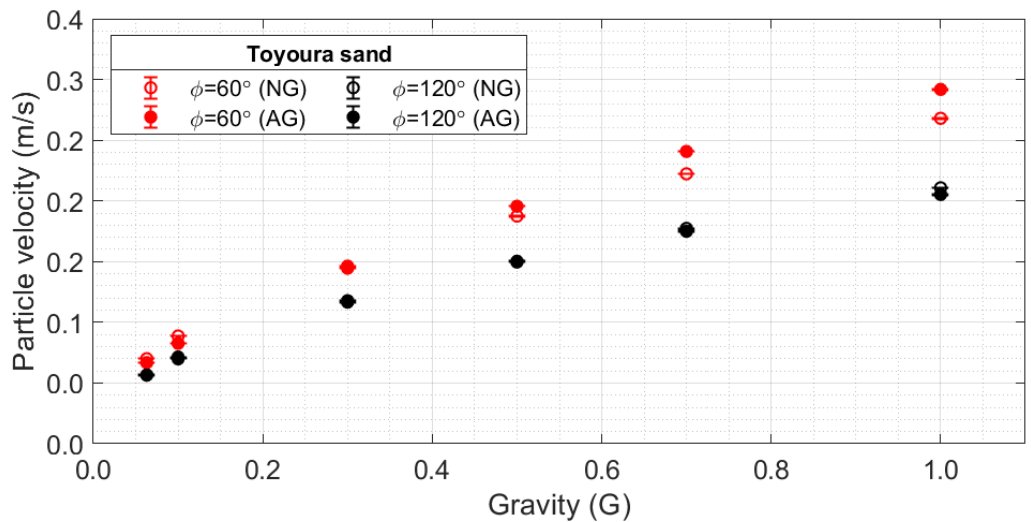

(c)

Figure 5: Gravity dependence of particle velocity just below the orifice obtained by DEM analysis under NG and AG conditions: (a) Alumina beads; (b) Silica sand No. 5; 
and (c) Toyoura sand. The particle velocity was measured at the centre position immediately below the orifice ( $x-y$ plane with $5 \mathrm{~mm} \times 15 \mathrm{~mm}$, and $1 \mathrm{~mm}$ length in the $z$ direction). The error bar denotes $95 \% \mathrm{CI}$. The measurement method and measured data of particle velocity are available in Supplementary Text 5. 

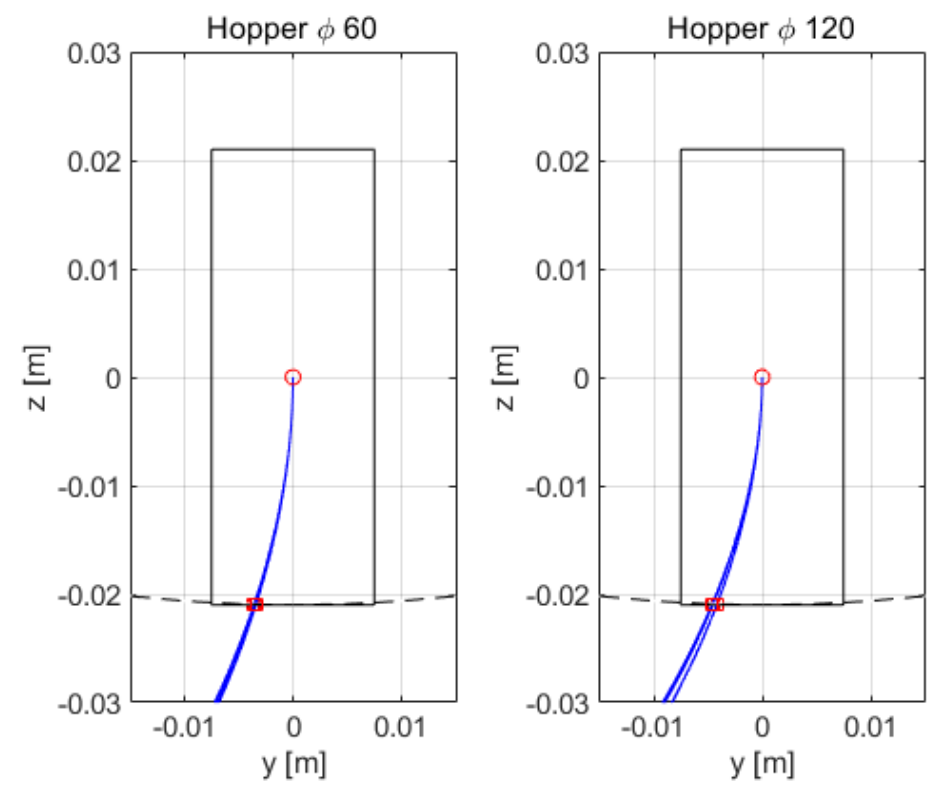

(a)

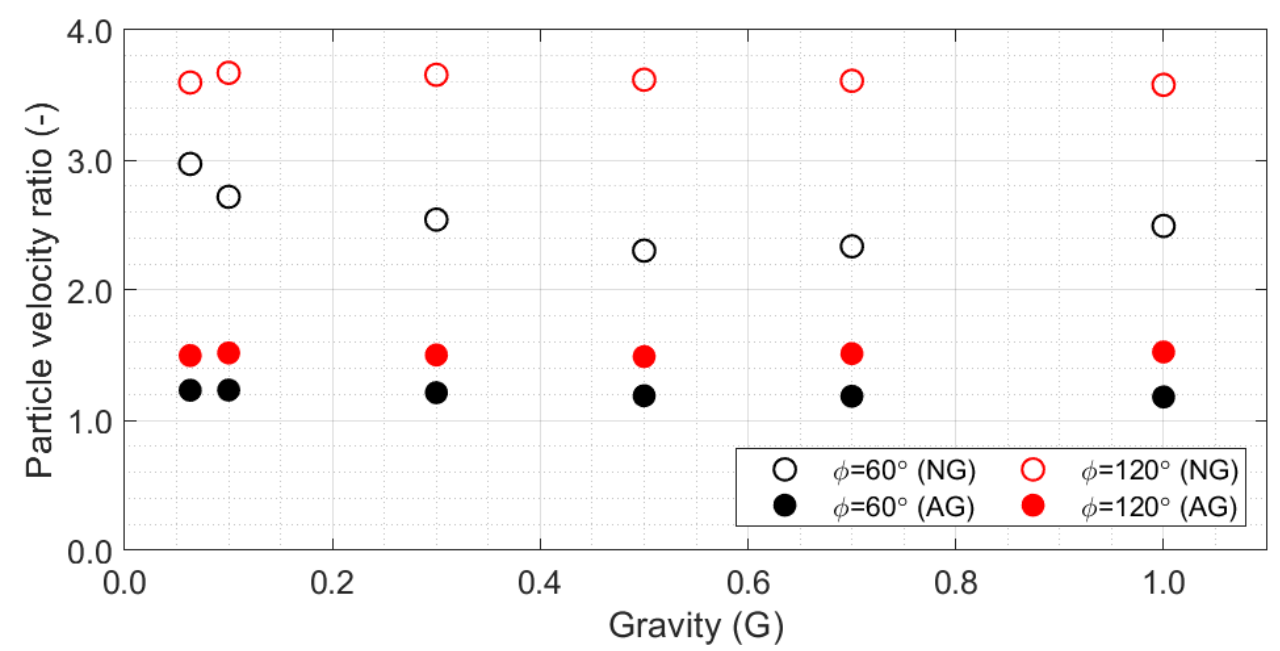

(b)

Figure 6: Particle motion of the alumina beads obtained by the equation of motion: (a) trajectories of the particles as seen from the $y-z$ plane of the hourglass under each AG condition; (b) ratio of the velocities at the bottom to the initial velocity. DEM analysis results are used for the initial velocities of particles just below the orifice. The solid symbol represents the data for the AG, whereas the open symbol represents that for the NG. 


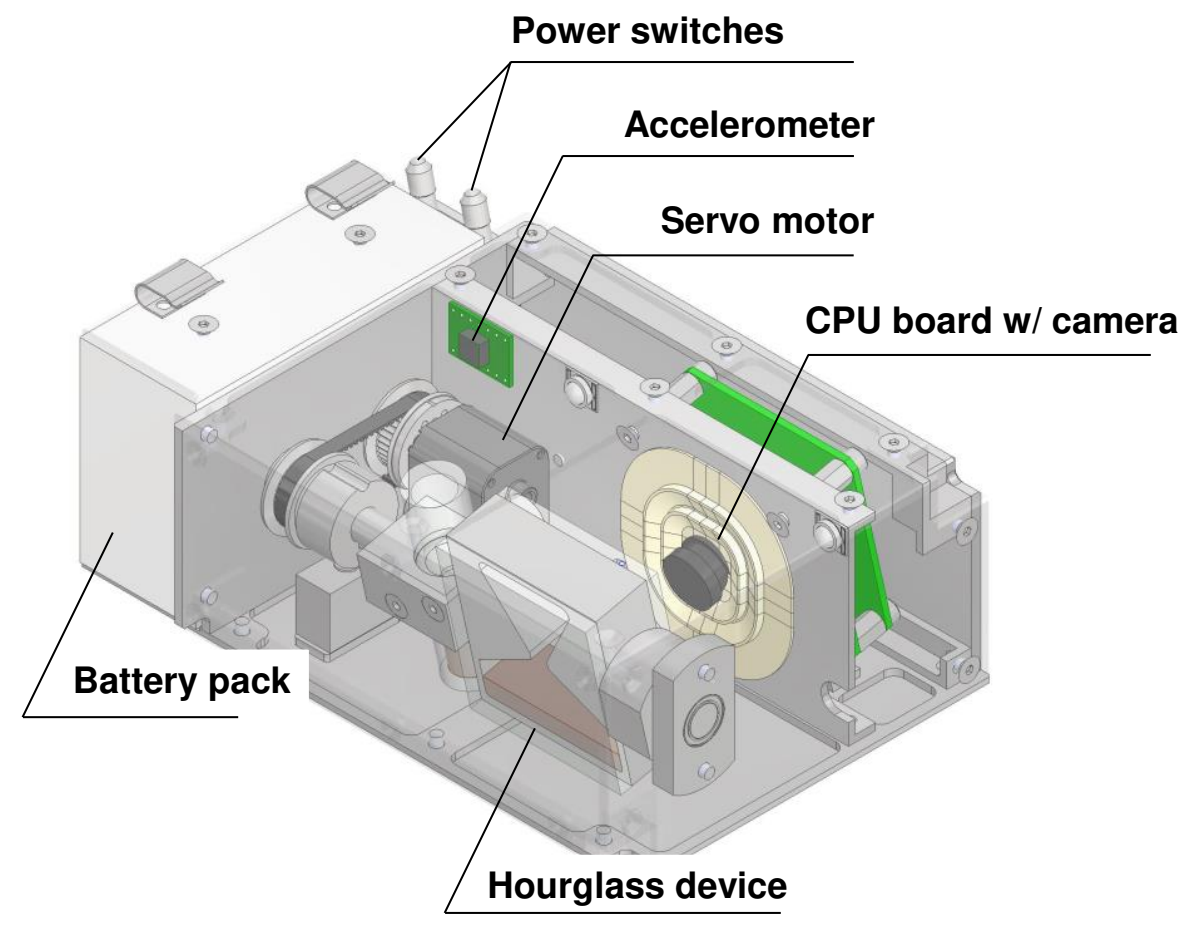

(a)
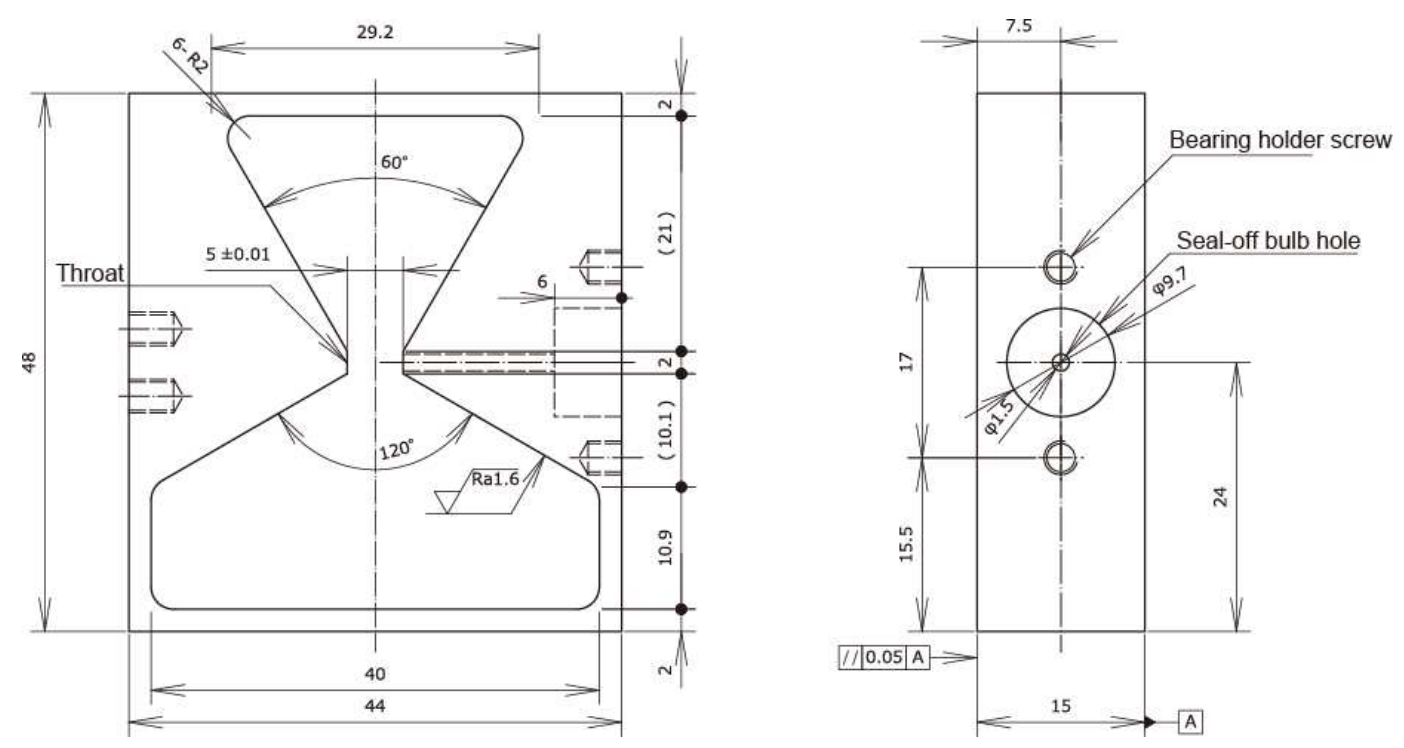

(b)

Figure 7: Schematic illustration of the experimental apparatus: (a) Component layout of the apparatus; (b) Specifications of hourglass shape (unit: $\mathrm{mm}$ ). 

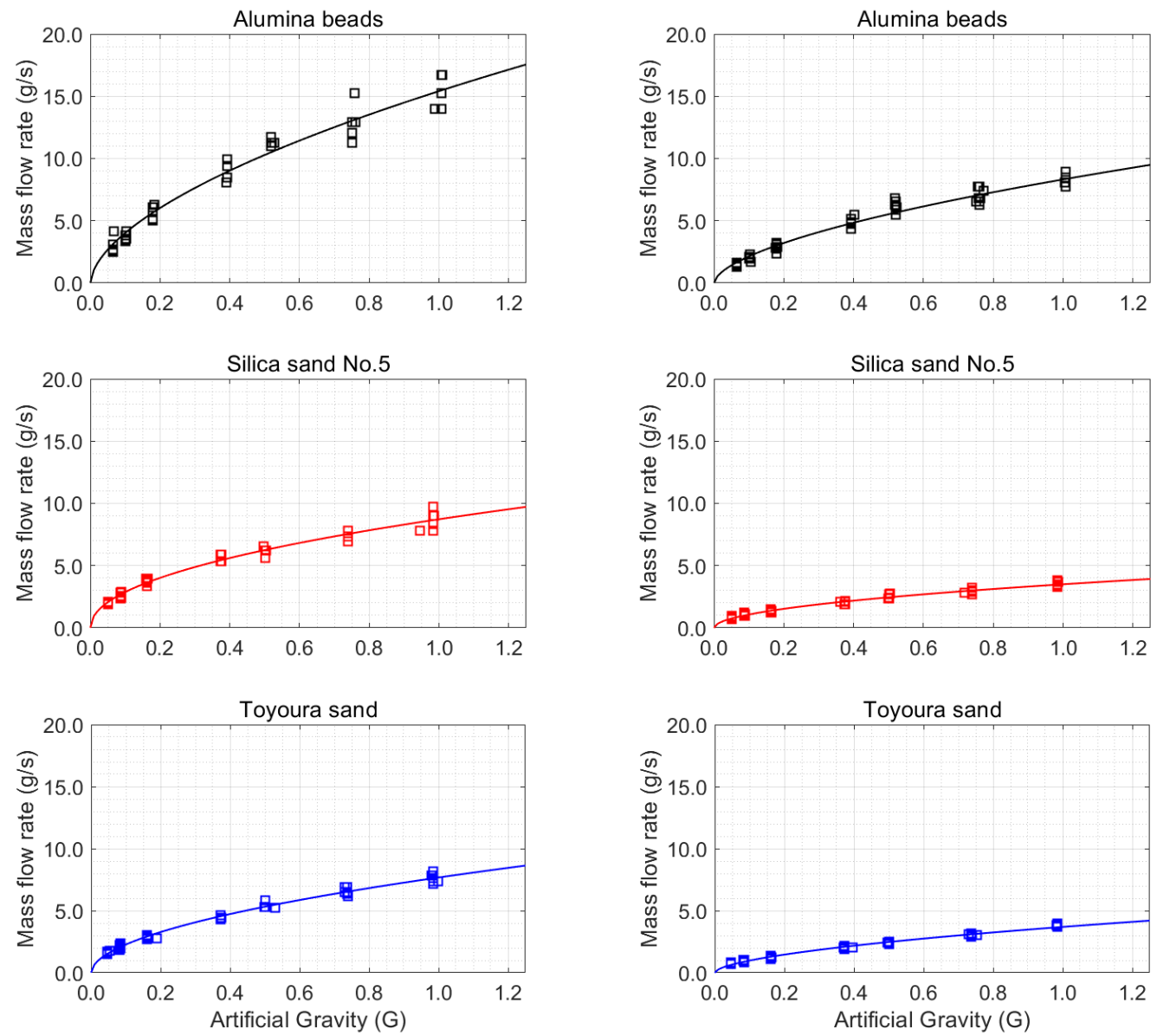

Hopper angle: 60 degrees

Hopper angle: 120 degrees

Figure 8: Regression analysis of the mass flow rate. 
Table 1. Snapshots of granular materials in microgravity. The hourglass image represents the final state of the flow when the granular media flows from the narrow area to the wide area (hopper angle is $60^{\circ}$ ) in $0.063 \mathrm{G}$.

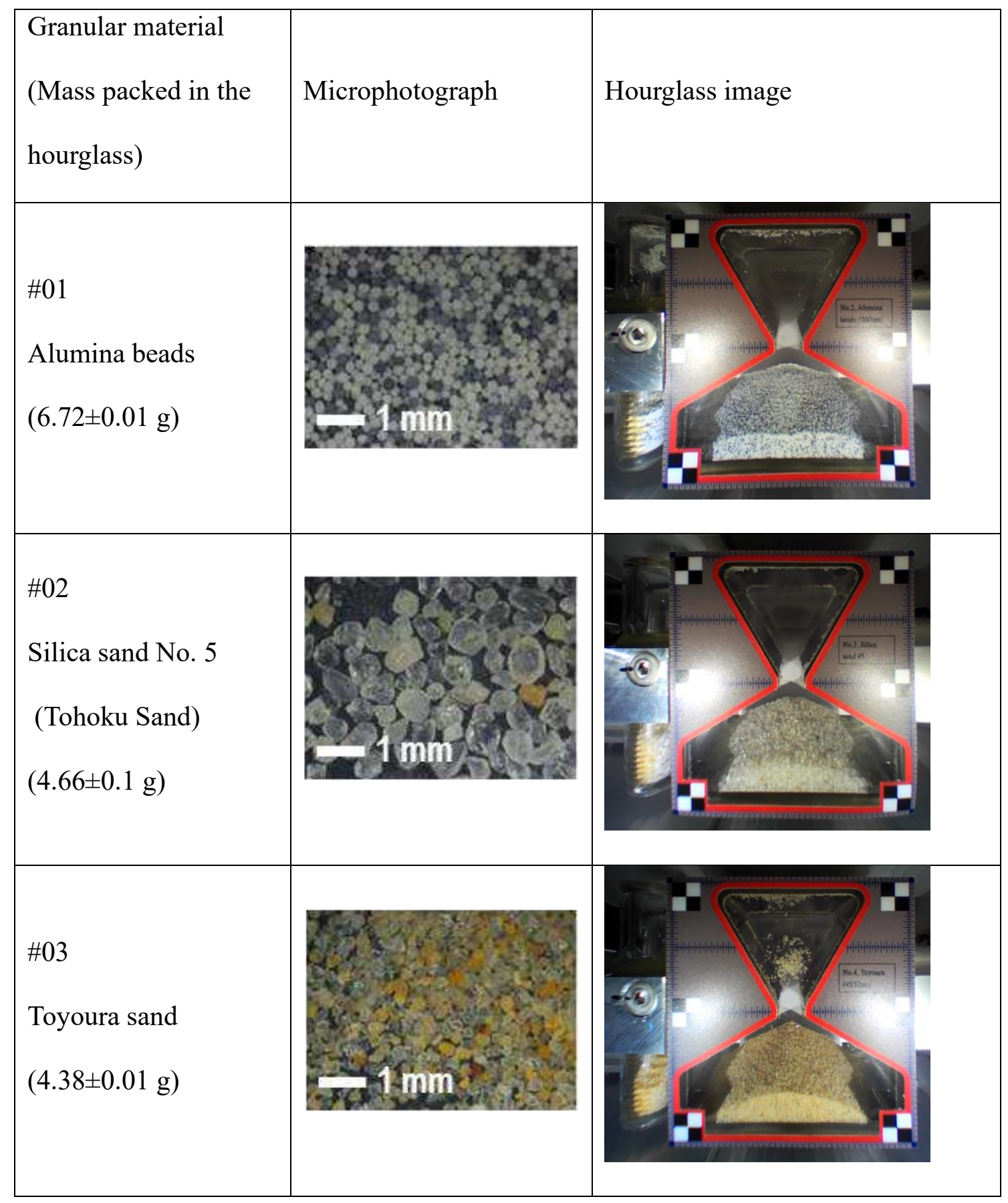




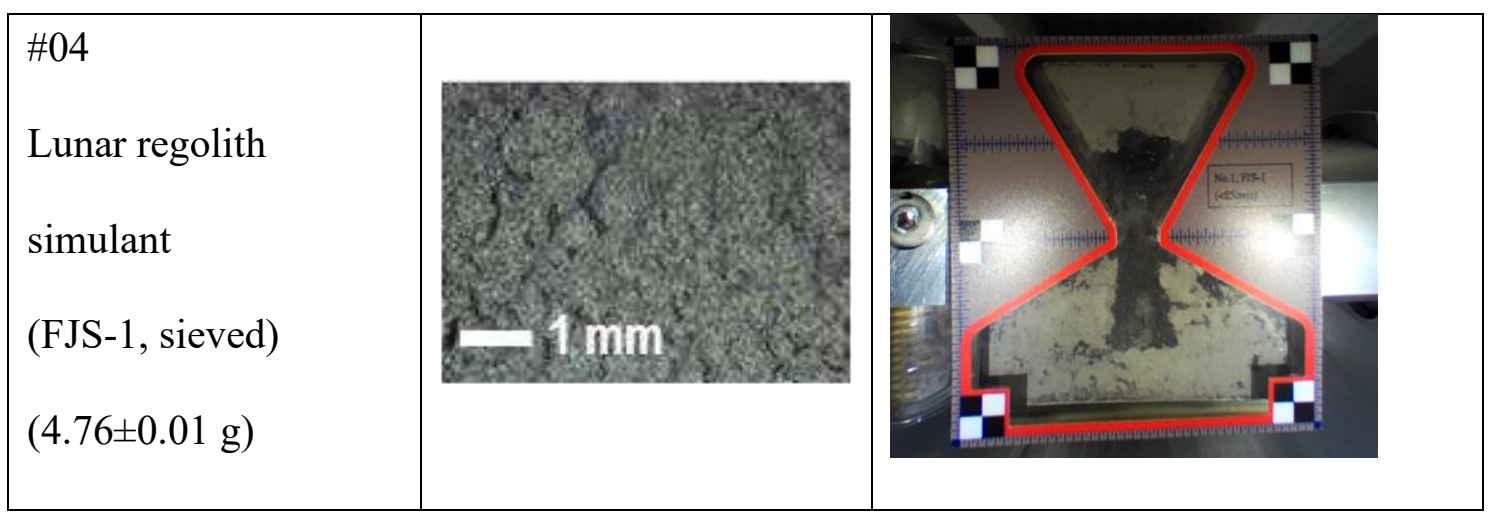

\begin{tabular}{|c|c|c|}
\hline $\begin{array}{l}\text { Granular material } \\
\text { (Particle size } \\
\text { distribution) } \\
\text { (Mass) }\end{array}$ & Microphotograph & Hourglass image \\
\hline $\begin{array}{l}\# 05 \\
\text { Phobos regolith } \\
\text { simulant } \\
(3.35 \pm 0.01 \mathrm{~g})\end{array}$ & & \\
\hline $\begin{array}{l}\# 06 \\
\text { Martian regolith } \\
\text { simulant } \\
\text { (JSC MARS-1A) } \\
(2.36 \pm 0.01 \mathrm{~g})\end{array}$ & & \\
\hline
\end{tabular}




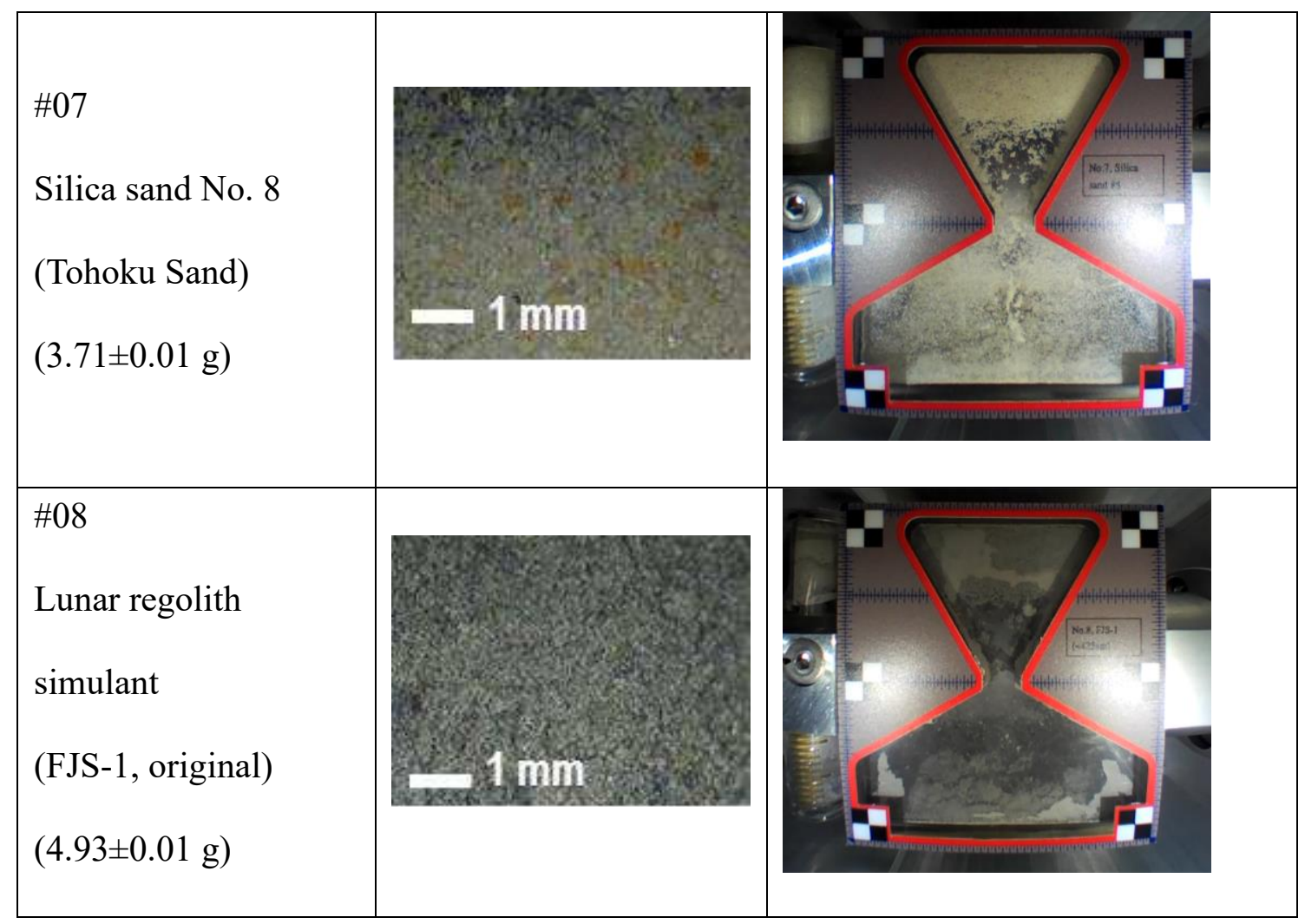


Table 2. Coefficients of the regression curves of the mass flow rate. Here, $R^{2}$ is the coefficient of determination of the regression curve.

\begin{tabular}{|l||l|c|c||c|c|c|}
\hline Hopper angle & \multicolumn{2}{|l|}{$60^{\circ}$} & \multicolumn{2}{l|}{$120^{\circ}$} \\
\hline \hline Granular media & $\alpha$ & $\beta$ & $R^{2}$ & $\alpha$ & $\beta$ & $R^{2}$ \\
\hline Alumina beads & 15.4 & 0.0836 & 0.972 & 8.32 & 0.0909 & 0.969 \\
\hline Silica Sand No. 5 & 8.73 & -0.0178 & 0.977 & 3.50 & 0.0144 & 0.975 \\
\hline Toyoura sand & 7.67 & 0.0289 & 0.989 & 3.69 & 0.0770 & 0.986 \\
\hline
\end{tabular}


Table 3: Parameters of Alumina beads, Silica sand No. 5, and Toyoura sand for DEM analysis.

\begin{tabular}{|c|c|c|c|}
\hline Particle & Alumina beads & Silica sand No. 5 & Toyoura sand \\
\hline Density $\left(\mathrm{g} / \mathrm{cm}^{3}\right)$ & 3.827 & 2.648 & 2.663 \\
\hline Young's modulus (MPa) & 5.0 & 5.0 & 5.0 \\
\hline Poisson's ratio (-) & 0.3 & 0.3 & 0.3 \\
\hline Rolling resistance (-) & 0.01 & 0.3 & 0.3 \\
\hline \multicolumn{4}{|l|}{ Particle-Particle } \\
\hline Static friction coefficient (-) & 0.30 & 0.50 & 0.50 \\
\hline Dynamic friction coefficient (-) & 0.30 & 0.50 & 0.50 \\
\hline Restitution coefficient (-) & 0.30 & 0.30 & 0.30 \\
\hline \multicolumn{4}{|l|}{ Particle-Hourglass } \\
\hline Static friction coefficient (-) & 0.33 & 0.22 & 0.18 \\
\hline Dynamic friction coefficient (-) & 0.33 & 0.22 & 0.18 \\
\hline Restitution coefficient (-) & 0.30 & 0.30 & 0.30 \\
\hline
\end{tabular}




\section{Supplementary Files}

This is a list of supplementary files associated with this preprint. Click to download.

- Supplementaryinformation.docx

- Supplementarymovie1.mp4

- Supplementarymovie2.mp4 